
\title{
25 Research Square \\ Cost-effectiveness of GeneXpert Compared to Smear Microscopy for Diagnosis of Tuberculosis in Ethiopia
}

\section{Abdene Kaso}

Addis Ababa University School of Public Health

Alemayehu Hailu ( $\nabla$ alemayehu.hailu@uib.no)

Addis Ababa University School of Public Health https://orcid.org/0000-0003-4872-8036

\section{Research article}

Keywords: Tuberculosis, Tuberculosis diagnosis, Cost-effectiveness, GeneXpert, smear microscopy, Ethiopia

Posted Date: July 29th, 2020

DOl: https://doi.org/10.21203/rs.3.rs-46904/v1

License: (c) This work is licensed under a Creative Commons Attribution 4.0 International License.

Read Full License 


\section{Abstract \\ Background}

Early diagnosis is one of the pillars of the TB (TB) control, and there are strong efforts to detect and treat cases in Ethiopia. Smear microscopy testing has been a routine test for the diagnosis of pulmonary TB in resource-constrained settings for a long time. Recently, many countries, including Ethiopia, are scaling up the use of GeneXpert without a precise evaluation of the cost and cost-effectiveness of this technology. Therefore, this study aimed to evaluate the cost-effectiveness of GeneXpert compared to smear microscopy tests for the diagnosis of TB patients in Ethiopia.

\section{Methods}

We develop a decision tree model using TreeAge Pro 2020 software. The model accounts for the prevalence and incidence of TB in the study area. The costs were estimated from the health providers' perspective in one year (in 2017/18). We applied an ingredients-based costing approach to identify, measure, and evaluate the smear microscopy cost and GeneXpert. We employed the 'proportion of cases detected' as an outcome measure of effectiveness. The incremental cost-effectiveness ratio (ICER) was calculated by dividing the changes in cost and change effectiveness. One way and probabilistic sensitivity analysis were done by varying different input parameters. All costs and ICER are reported in 2018 US\$.

\section{Results}

The unit cost per test for GeneXpert and smear microscopy testing was US\$12.9 and \$3.1, respectively. The average cost of testing using GeneXpert was $\$ 113.0$ and $\$ 3.3$ for smear microscopy. The cost of the cartridge (\$10.7) was the primary (83\%) parameter influencing the overall cost of GeneXpert, while it was the cost of reagent and consumables $\$ 1.28(41 \%)$ for the smear microscopy. The ICER for GeneXpert strategy was $\$ 729.8$ per new TB cases detected compared to smear microscopy. The sensitivity analysis indicates that TB prevalence was the most influential parameter on the ICER.

\section{Conclusion}

The present study indicates that testing all individuals with suspected TB using GeneXpert is a very costeffective strategy compared to smear microscopy, and therefore, it can be part of the routine diagnostic testing strategy in Ethiopia.

\section{Background}


Tuberculosis (TB) is one of the significant challenges globally and in Ethiopia. The 2016 global burden of disease (GBD) study estimate that TB is responsible for 1 out of 4 Years Lived with Disability (YLD) and 1.3 million deaths annually. About $60 \%$ of the estimated number of cases occurred in Asia and Africa. Following the launching of the Directly Observed Treatment Short Course (DOTS) in 1990, the global prevalence and mortality of TB decreased substantially. It was estimated that 37 million lives were saved between 2000 and 2013 through effective diagnosis and treatment. However, TB incidence in Africa continues to be high, and Ethiopia is one of the high TB burden countries. For instance, in 2016, 163 new TB cases were reported per 100,000 populations. From 2005 to 2015, the prevalence of Multiple DrugResistant TB (MDR-TB) in Ethiopia has increased from 1.6-2.7\% in new cases and 11.8 to $14.0 \%$ in retreated TB cases [1-5].

Early diagnosis and treatment of cases are among the pillars of the National TB Control Program (NTCP) in Ethiopia, and there is a need for an efficient and sensitive diagnostic tool to detect and treat the infection [6]. For a long time, TB diagnosis relies on smear microscopy [7]. Smear microscopy has a sensitivity of about $70 \%$, and it cannot detect MDR-TB from a patient sample. The low sensitivity of smear microscopy can delay diagnosis and lead to the secondary spread of TB infection [8]. Therefore, there is a critical need to apply new diagnostic methods that are highly sensitive and can also detect MDR-TB strains [9].

GeneXpert is more sensitive than smear microscopy in detecting TB, with culture as a gold standard [10, 11]. According to the WHO report, GeneXpert has $89 \%$ sensitivity and $99.7 \%$ specificity, and therefore, it can increase TB case finding from $72-85 \%$ to $95-99 \%$ compared to the smear microscopy. A national validation study reports that the overall sensitivity and specificity of GeneXpert for detecting MDR-TB was nearly $100 \%$ in new cases, whereas it was about $95 \%$ and $90 \%$ in retreated cases. GeneXpert technology is highly recommended in populations with a high MDR-TB burden like Ethiopia [12]. The overall sensitivity and specificity of GeneXpert in detecting MDR cases were $96.8 \%$ and $92.9 \%$, respectively [13]. GeneXpert also has some other advantages: it is easy to train health workers in its use, no risk of sample cross-contamination, and no need for a specific biological safety environment [14].

Many countries, including Ethiopia, are scaling up the use of GeneXpert without evaluating the tests' cost and cost-effectiveness. Recently, the Ethiopian Federal Ministry of Health $(\mathrm{FMOH})$ recommended routine testing using GeneXpert at facilities having less than 12 tests per day. However, the scale-up of GeneXpert as a diagnostic test in a routine healthcare delivery setting might have substantial economic implications. For example, a study shows that the diagnosis of TB among suspected individuals can increases the costs of testing from US $\$ 28-\$ 49$ to $\$ 137-\$ 151$ per TB case detected when GeneXpert is used instead of smear microscopy [15]. The absence of information on cost and cost-effectiveness analysis of this new technology on routine patients hinders policymakers from making decisions to scale up the services to all facilities in the country. Therefore, this research aimed to evaluate the cost and costeffectiveness of the GeneXpert compared to smear microscopy method for the diagnosis of TB suspected individuals as routine diagnostic testing. 


\section{Methods}

\section{Study design and setting}

In this cost-effectiveness analysis, we employed a decision analysis model to compare the two TB diagnostic methods (GeneXpert and smear microscopy). The costing part of this study was conducted using an institutional-based cross-sectional study design. A costing study was conducted in selected Health Centers and Hospitals in Arsi Zone from January to February 2019. The Arsi Zone is located about $175 \mathrm{~km}$ away from Addis Ababa, and it comprises 28 woredas (districts) and two town administration. The zone consists of a total of 7 hospitals and 102 health centers. Diagnoses of TB cases were conducted using GeneXpert in four hospitals only while smear microscopy was conducted in all health centers and Hospitals.

\section{Description of the interventions}

We compared two diagnostic methods: smear microscopy and GeneXpert methods. In the GeneXpert methods, a single sputum specimen was tested for all persons suspected of TB. The test procedure was used on fresh sputum samples or other samples. The sputum sample was combined with the reagent, in a 2:1 ratio for sputum liquefaction and inactivation. Then it was incubated at room temperature for 1520 minutes. After incubation, a total of $2 \mathrm{ml}$ of the mixture was introduced into a GeneXpert cartridge, loaded into a GeneXpert instrument for analysis. The instrument generates the test report automatically within 2 hours [16]. In the smear microscopy method, all persons were presented with spot-spot sputum for TB diagnosis.

\section{Model structure}

A decision analysis model was developed using TreeAge Pro software version 2019 (Fig. 1). This economic evaluation was conducted with 1332 suspected TB patients per diagnostic method. TB prevalence was used as the probability of being positive for each testing method. A positive test result might be either a Test Positive (True Positive) or a Test Negative (False Positive) based on the diagnostic techniques' sensitivity. A negative test result might also be either a Test Negative (True Negative) or a Test Positive (False Negative) based on the specificity of the diagnostic method.

\section{Identification of cost}

Identification, measurement, and valuation of the cost of TB diagnosis techniques were conducted from the health providers' perspective. All cost for diagnosis of TB suspected individuals with smear microscopy, and the GeneXpert technique was included. For smear microscopy, different costs like building space, equipment, overhead, staff, and training were included in cost-effectiveness analysis. Besides, the cost of quality control conducted every week, and opening new batch reagents were included. However, the cost of the microscope's annual calibration was not included in the study due to the absence of data. 
For GeneXpert, the costs of building space, overhead, GeneXpert IV module machine, and accessories were included. Moreover, the cost of operating staff and training needed for conducting GeneXpert diagnosis were included in the analysis. Costs of External Quality Control (EQC) and annual calibration of the GeneXpert module were also accounted for in the analysis. However, for both TB diagnostic methods, the cost of annual service maintenance was not included due to the absence of cost data.

\section{Measurement of cost}

We collected overhead costs, building space, equipment, staff, reagents, and consumables related to each technique from procurement invoice, expert and managers opinion, a systematic review, and administration reports available in the health facilities. The cost types and quantity of each resource used in each diagnostic technique were recorded in the Microsoft Excel 2010 spreadsheet.

\section{Valuation of cost}

Cost estimation for compared diagnostic methods was estimated for a one-year time frame using an ingredients approach method. The cost of reagent and consumables for smear microscopy was obtained by dividing the gross cost of a given measure of each reagent over the average number of tests that can be performed using that amount. Besides, the cost of the cartridge for the GeneXpert test was obtained from a published source [17]. The cost of overhead for each technique was calculated by taking $5 \%$ of the total health overhead cost based on expert opinion and discussion with laboratory head. For buildings, an expected lifetime of 30 years was used. Laboratory space cost was allocated based on the proportional size required for conducting TB diagnosis. The cost of quality control (QC) for smear microscopy was obtained by identifying all resources needed to perform QC and multiplying by the total QC recommended according to guidelines and SOP in the year. The cost of EQC for GeneXpert was obtained by multiplying the cartridge cost by the amount of cartridge used in four-term proficiency testing. GeneXpert module annual calibration must be performed every 2000 tests or every 12 months. The cost of the annual calibration was obtained from the Stop TB partnership. The cost of EQC per test was obtained by dividing the annual calibration cost over 2000 tests, as recommended by the manufacturer when QC should be performed [18]. The cost of equipment for each diagnostic method was obtained by dividing the equipment's annualized cost over the number of tests performed. The useful life of microscopy was assumed to be ten years and run five tests per day. Based on expert opinion and observation, about $100 \%$ and $40 \%$ of microscopy use were allocated for smear microscopy at hospitals and health centers.

Concerning the GeneXpert testing, the laboratory utilized four modules GeneXpert machine operating four simultaneous tests and running for 8 hours per day. The instrument was assumed to have a useful life of 10 years and to process, on average, eight sputum samples per day. We valued personnel cost based on an estimated proportion of working time spent on TB diagnosis by each method. The smear microscopy diagnostic procedure assumed to take 35 , starting from sputum smear preparation to manual slide reading. Moreover, we assumed that all procedures related to the GeneXpert method would take 25 minutes per test. The number of training days for conducting tests by AFB smear microscopy and GeneXpert was assumed five days and three days. Data on the cost of training was obtained from 
OPHRCBQAL (Oromia Public Health Research Capacity Building \& Quality Assurance Laboratory). Capital costs were annualized using a standard discount rate of $3 \%$ per year as used in similar studies $[19,20]$. Local costs were collected in Ethiopian birr and converted to United States Dollars (US\$) using exchange rates by the Commercial Bank of Ethiopia (1US\$ = ETB 27.18) [21]. All the costs were adjusted for inflation using the consumer price index of the year 2018 as a base year cost.

\section{Model parameters and effectiveness}

The effectiveness parameters in this analytic decision model were based on the prevalence of TB and the diagnostic accuracy of the diagnostic methods. Input for each method was obtained from the published study on the diagnostic accuracy of the methods and TB prevalence. We used the pooled values as the base estimates and the $95 \%$ confidence interval $(\mathrm{Cl})$ values as the upper and lower limits for the tests' diagnostic accuracy. For TB prevalence, we took $+/-50 \%$ of the average value as upper and lower bounds. For cost data, we assumed the lower bound and the upper bound to be $+/-20 \%$ of the base case values.

Table 1

Parameters used in the decision analysis model

\begin{tabular}{|llllll|}
\hline Model input & Base value & Min & Max & SD & Sources \\
\hline Test sensitivity GeneXpert & 0.88 & 0.84 & 0.92 & 0.02 & {$[13,16]$} \\
\hline Test specificity GeneXpert & 0.99 & 0.97 & 1.00 & 0.01 & {$[13,16]$} \\
\hline Test sensitivity of two smear microscopy & 0.79 & 0.52 & 0.82 & 0.13 & {$[38-40]$} \\
\hline Test specificity of Two smear microscopy & 0.97 & 0.94 & 0.99 & 0.01 & {$[38-40]$} \\
\hline TB prevalence & 0.16 & 0.08 & 0.24 & 0.04 & {$[41]$} \\
\hline Cost of smear microscopy test negative (US\$) & 3.09 & 2.48 & 3.72 & 0.31 & Primary \\
\hline Cost smear microscopy test positive (US\$) & 55.84 & 44.67 & 67.01 & 5.80 & Primary \\
\hline Cost of GeneXpert test positive (US\$) & 77.84 & 62.30 & 93.46 & 7.79 & Primary \\
\hline Cost of GeneXpert test negative (US\$) & 12.92 & 10.34 & 15.50 & 1.29 & Primary \\
\hline
\end{tabular}

\section{Data collection}

The cost data were collected from selected eight health facilities. The data collection tools were developed based on the guidelines for cost and cost-effectiveness analysis of TB control prepared by $\mathrm{WHO}$ in 2002. After the pretest was conducted, necessary correction was made on data collection tools. The data collection process was coordinated and supervised by a hired supervisor and the principal investigator. Data was collected from the procurement invoice, patient log book, payrolls of salary, administration report, literature review, and estimates of expert opinion and other responsible bodies.

Trained laboratory technicians collected data after giving two-day training on data collection format. The extracted data were cross-checked using the TB register, and the principal investigator checked completeness of collected data. 


\section{Cost-effectiveness analysis}

The model's primary outcome measure was the proportion of positive cases detected when each diagnostic method was used for TB diagnosis in suspected TB individuals. Cost and effectiveness data were entered and analyzed using Microsoft Excel 2016 spreadsheet. TreeAge Pro version 2019 software was used to build the decision tree model for cost-effectiveness and sensitivity analyses.

An analytic decision model was developed using TreeAge Pro software version 2019 to estimate the costeffectiveness of the routine diagnostic methods for TB's diagnosis. A cost-effectiveness analysis was performed by putting the diagnostic accuracy, TB prevalence, the proportion of case detected, and costs of each method into the software. The diagnostic method had payoff one for case detected, while cases that were not detected had a payoff of zero. In all diagnostic techniques, the cost payoffs were the costs for the diagnostic method for TB diagnosis. Since there is no widely accepted Willingness to pay (WTP) threshold for this intermediate outcome (i.e., per case detected), we did not use any threshold, and only estimated ICER was presented in this study.

\section{Sensitivity analysis}

We performed a one-way sensitivity analysis based on input parameters used in the model using the minimum and maximum values obtained from a literature review. A tornado diagram was used to illustrate the influence of model parameters based on the magnitude of their effects on the ICER. Besides, we conducted a scenario analysis for low and high values of parameters used in the model. We also conducted Probabilistic Sensitivity Analysis (PSA) for the distribution of selected variables using Monte Carlo simulation with 100,000 iterations. The model parameters were specified as a distribution and varied to see what difference they created on ICER. The result of the analysis was presented using costeffectiveness acceptability frontiers, and cost-effectiveness scatters plot. Gamma distribution was used for cost, while beta distribution was utilized for probabilities.

\section{Results}

Among 1,332 study participants diagnosed with smear microscopy, 692 (52\%) were male, and 640 (48\%) were female. Out of the total patients diagnosed by smear microscopy, 1258(94.4\%) had smear-negative results, while 74 (5.6\%) had smear-positive. About 724 (54.4\%) males and 608 (45.6\%) females were tested by the GeneXpert method. Out of patients diagnosed by the GeneXpert technique, $221(16.6 \%)$ had TB, while about 1,111 (83.4\%) were negative for TB (Table 1). 
Table 2

Characteristics of TB suspected patients by testing techniques

\begin{tabular}{|lll|}
\hline Category & $\begin{array}{l}\text { Smear microscopy } \\
\text { Frequency (\%) }\end{array}$ & $\begin{array}{l}\text { GeneXpert } \\
\text { Frequency (\%) }\end{array}$ \\
\hline Male & $692(52.0 \%)$ & $724(54.4 \%)$ \\
Female & $640(48.0 \%)$ & $608(45.6 \%)$ \\
Test positive & $74(5.6 \%)$ & $221(16.6 \%)$ \\
Test negative & $1,258(94.4 \%)$ & $1,111(83.4 \%)$ \\
\hline
\end{tabular}

\section{The unit cost of GeneXpert and smear microscopy methods}

The unit cost of smear microscopy and GeneXpert methods varies from facility to facility. The average unit cost of the test in the smear microscopy group was $\$ 3.3$ (ranging from $\$ 2.4$ to $\$ 4.96$ based on the annual volume of testing and level of health facilities). The unit cost of the GeneXpert method was $\$ 12.69$ and $\$ 13.22$ for high and low volume testing health facilities (Table 3).

Table 3

Unit cost of smear microscopy and GeneXpert diagnostic method in Arsi zone, Ethiopia, 2019

\begin{tabular}{|llllll|}
\hline Health facilities & Supplies & $\begin{array}{l}\text { Overhead and } \\
\text { space }\end{array}$ & Equipment & $\begin{array}{l}\text { Staff and } \\
\text { training }\end{array}$ & $\begin{array}{l}\text { Unit } \\
\text { cost }\end{array}$ \\
\hline $\begin{array}{l}\text { Smear microscopy } \\
\text { sites }\end{array}$ & & & & & \\
\hline Bokoji HSP & $\$ 312.30$ & $\$ 106.74$ & $\$ 210.70$ & $\$ 265.54$ & $\$ 2.79$ \\
\hline Bokoji HC & $\$ 240.98$ & $\$ 59.32$ & $\$ 64.58$ & $\$ 190.29$ & $\$ 3.32$ \\
\hline Kersa HSP & $\$ 214.14$ & $\$ 103.34$ & $\$ 151.88$ & $\$ 170.16$ & $\$ 4.96$ \\
\hline Sagure HC & $\$ 312.30$ & $\$ 59.39$ & $\$ 67.95$ & $\$ 189.95$ & $\$ 2.40$ \\
\hline Gobesa HSP & $\$ 271.97$ & $\$ 103.28$ & $\$ 188.01$ & $\$ 192.33$ & $\$ 3.62$ \\
\hline Meraro HC & $\$ 285.58$ & $\$ 58.21$ & $\$ 66.77$ & $\$ 183.61$ & $\$ 2.68$ \\
\hline GeneXpert sites & & & & & \\
\hline Asella HSP & $\$ 8,002.7$ & $\$ 130.18$ & $\$ 882.18$ & $\$ 543.37$ & $\$ 12.69$ \\
\hline R/Didea HSP & $\$ 6,204.2$ & $\$ 102.06$ & $\$ 879.11$ & $\$ 466.12$ & $\$ 13.22$ \\
\hline Note: HC: Health center, HSP: Hospital & & & \\
\hline
\end{tabular}


Costs per test conducted were $\$ 12.9$ for GeneXpert and $\$ 3.1$ for smear microscopy, respectively. The smear microscopy costs were attributable primarily to consumables $\$ 1.3(41.3 \%)$. Similarly, the main drivers (82.6\%) of GeneXpert's unit cost were the GeneXpert cartridges ( $\$ 10.7$ per test). Overhead costs associated with performing GeneXpert were low ( $\$ 0.17$ per test) compared to smear microscopy (\$0.37 per test) (Fig. 2).

\section{Cost per TB case detected}

The cost per TB case detected for smear microscopy diagnostic method was $\$ 55.8$. The cost of consumable accounts for $\$ 23.0$ (41.1\%) and the cost of staff accounts for $\$ 16.1$ (28.9\%) of the total cost. Overhead and equipment cost accounts for $30.01 \%$ of the total unit cost. Moreover, the cost per TB case detected for the GeneXpert testing was \$77.9. A higher percentage is attributed to the cost of consumable (cartridge) \$64.3 (82.5\%) and equipment \$7.97 (10.23\%) (Fig. 3).

\section{Scenario analysis of the costs}

In the smear microscopy diagnostic method, reducing the useful life of the capital equipment from ten to five years increased the cost of the test to \$3.6. If the number of tests performed per day increased to ten, the test's cost would decrease by $16.13 \%$. Furthermore, allocating $100 \%$ of microscopy use at health centers to smear microscopy increased the cost of the test to \$3.3.

In the GeneXpert technique, reducing the cartridge price by $10 \%$ and increasing the number of tests performed per day to sixteen from eight lowered the unit cost of GeneXpert per test to US\$ 11.66 and $\$ 11.9$, respectively. However, reducing the useful life of the GeneXpert machine and another item to five years increased the cost of GeneXpert by $7.0 \%$ (Table 4 ).

Table 4

A scenario analysis of cost assumptions

\begin{tabular}{|c|c|c|c|}
\hline Parameter & $\begin{array}{l}\text { Unit cost } \\
\text { per test }\end{array}$ & $\begin{array}{l}\text { Change } \\
(\%)\end{array}$ & $\begin{array}{l}\text { Cost per case } \\
\text { detected }\end{array}$ \\
\hline \multicolumn{4}{|l|}{ Smear microscopy (base-case unit cost $=\$ 3.1$ ) } \\
\hline $\begin{array}{l}\text { Reduce the useful life of capital equipment from } 10 \text { to } \\
5 \text { years }\end{array}$ & $\$ 3.59$ & +15.8 & $\$ 64.6$ \\
\hline Increase the number of tests to 10 per day & $\$ 2.60$ & -16.1 & $\$ 46.7$ \\
\hline $\begin{array}{l}\text { Allocate } 100 \% \text { of microscopy to smear microscopy at } \\
\text { the health center }\end{array}$ & $\$ 3.32$ & +7.1 & $\$ 59.7$ \\
\hline \multicolumn{4}{|l|}{ GeneXpert (base-case unit cost $=\$ 12.92$ ) } \\
\hline $\begin{array}{l}\text { Reduce the useful life of capital equipment from } 10 \text { to } \\
5 \text { years }\end{array}$ & $\$ 13.83$ & +7.0 & $\$ 83.4$ \\
\hline Increase the number of tests to 16 per day & $\$ 11.87$ & -8.1 & $\$ 71.6$ \\
\hline Reduce the price of the cartridge by $10 \%$ & $\$ 11.66$ & -9.8 & $\$ 70.3$ \\
\hline
\end{tabular}




\section{Cost-effectiveness ratio}

During CEA, the GeneXpert strategy has a high expected number of cases detected and expected cost compared to the smear microscopy. The expected cost was $\$ 22.2$ for the GeneXpert method while it was $\$ 11.1$ for smear microscopy. As a result, the GeneXpert diagnostic method's ICER was $\$ 729.8$ per proportion of cases detected compared to AFB smear microscopy (Table 5).

Table 5

Incremental cost-effectiveness ratios of GeneXpert compared to smear microscopy

\begin{tabular}{|c|c|c|c|c|c|c|}
\hline Interventions & Cost & Inc. Cost & Effectiveness & Inc. Effectiveness & ICER & ACER \\
\hline Smear microscopy & 11.1 & Ref. & 0.009 & Ref. & Ref. & 1218.9 \\
\hline GeneXpert & 22.2 & 11.14 & 0.024 & 0.015 & 729.82 & 912.4 \\
\hline
\end{tabular}

\section{One-way sensitivity analysis}

One-way sensitivity analysis for TB diagnostic methods was presented using a tornado diagram. In a one-way sensitivity analysis, the prevalence of TB was a vital driver of the ICER of the GeneXpert. The ICER increases from $\$ 530.57$ to $\$ 1401.13$ per proportion of cases detected for TB prevalence from 0.24 to 0.08 . Other variables with a moderate influence on the model were the specificity and sensitivity of the GeneXpert diagnostic methods. The specificity of GeneXpert ranges from 97-100\%, and the ICER would range from $\$ 659.72$ to $\$ 739.86$. Moreover, the ICER ranges from $\$ 756.50$ to $\$ 706.69$ for GeneXpert sensitivity ranges from 0.84 to 0.92 (Fig. 4).

The incremental proportion of TB cases detected, comparing the GeneXpert method to smear the prevalence of TB infection, mostly influenced the microscopy algorithm. The incremental proportion of cases detected ranges from 7 per 1000 TB suspects to 23.6 per 1000 TB suspects for TB prevalence of 8-24\% among TB suspects. However, as the GeneXpert diagnostic method's specificity increases from $97-100 \%$, the incremental effectiveness declines from 19.1 per 1000 TB to 14.8 per 1000 TB suspects (Fig. 5).

\section{Probabilistic sensitivity analysis output}

A hundred thousand iterations of the PSA showed that the ICER of the GeneXpert algorithm was around the baseline value. The ICER of the GeneXpert algorithm was $\$ 726.8$ per proportion of TB cases detected. The PSA results found that at the WTP threshold of, for example, $\$ 1000$ per Tb case detected, the probability of the GeneXpert diagnostic being cost-effective method is about $90 \%$ (Fig. 6).

\section{Discussion}


TB is a public health threat despite the availability of advanced diagnostic tools [22]. In order to successfully control the spread of MTB, cases must be detected and treated immediately. GeneXpert is one of the advanced diagnostic tools enabling POC for same-day diagnosis and treatment[23]. This study aimed to evaluate the cost-effectiveness of GeneXpert compared to smear microscopy using an analytic decision model. An ingredient-based costing approach was employed. In this study, the unit cost per test for smear microscopy diagnostic technique was $\$ 3.1$, while the unit cost per case detected was US\$ 55.5.

Moreover, the unit cost per test was high for the GeneXpert-based algorithm compared to the conventional smear microscope technique. This is consistent with studies conducted in Brazil and India $[24,25]$ and South Africa [26]. This relatively high cost might be due to expensive equipment and cartridge and high maintenance costs required by the GeneXpert algorithm compared to the routine smear microscopy method.

In this study, most drivers of the unit cost of GeneXpert were cartridge and consumable cost $(80 \%)$. Moreover, the study result indicated that if the cost of cartridge reduced by $10 \%$, the unit cost would reduce by $9.75 \%$ below the base case. This estimate was similar to a study conducted in South Africa (47\%) [19] and Uganda [27] that indicated the most of the costs of GeneXpert were attributed to the high price of the cartridge. This estimate shows that the high cost of cartridges can be the major obstacle to the full implementation of the diagnostic method as a routine test. Therefore, for the full scale-up of this technology, controlling financial sustainability by either increasing TB funding or reductions in cartridge price is needed, as indicated in another study [28].

In this study, the cost of smear microscopy and GeneXpert in low testing volume health facilities was $\$ 4.96$ and $\$ 13.22$. Our scenario analysis result also revealed that the cost of both diagnostic algorithms was reduced from the base case estimate by increasing the volume of tests per day. This cost estimate is in line with finding from sub-Sahara Africa [29] and Uganda [30] on the cost and cost-effectiveness of the GeneXpert diagnostic test. The cost of the test method was high in health facilities where their testing volume was low. The most probable reason was the decrease in cost capital equipment as testing volume per day increased.

The control of TB is still the problem of developing countries due to the rise of MDR-TB and poor case detection. This forced countries to raises their concern about using advanced laboratory diagnostic methods [31]. However, advanced technology should have an acceptable cost and cost-effectiveness to be used as a routine diagnostic procedure. Our study result demonstrated that GeneXpert testing among patients with suspected TB is very cost-effective. This cost estimate is in line with a study in China [32] and the United States [33] that found incorporating GeneXpert in the TB diagnostic algorithms was highly cost-effective.

Moreover, this study's result was consistent with a study done in South Africa [34] that found that using a novel diagnostic test (GeneXpert) for TB diagnosis was cost-saving and cost-effective. However, this finding contradicted the study in Uganda that found GeneXpert was not cost-effective [27]. This 
difference might be due to the algorithm compared with GeneXpert as MODS has almost similar diagnostic accuracy and low cost compared with the GeneXpert diagnostic method.

The GeneXpert technique's cost-effectiveness was indicative of the potential use of this method for the routine diagnosis of TB. However, this advanced technique's cost-effectiveness depends on the prevalence of TB and the diagnostic accuracy of compared algorithms. In one way, sensitivity analysis, the ICER of GeneXpert, was more sensitive to the prevalence of TB: As the prevalence of TB increases, the GeneXpert algorithm becomes the best optimal strategy despite the higher cost. Besides, the ICER of GeneXpert was moderately influenced by the specificity and sensitivity of GeneXpert diagnostic methods. Similarly, studies on the cost-effectiveness of GeneXpert indicated that the most driver of ICER of GeneXpert was the prevalence of TB $[35,36]$. On the other hand, other studies result demonstrated that the diagnostic accuracy of GeneXpert was the most influential parameter on the ICER of this test [6,37]. If the GeneXpert was used at high TB prevalent areas, the probability of GeneXpert to detect more cases might increase, and this algorithm could be an optimal strategy.

Even though this study is the first of its kind measuring the cost-effectiveness of TB diagnostic method in Ethiopia, it has some limitations. First, the use of 1-times or 3-times GDP per capita per DALY averted, as WTP to decide the cost-effectiveness of the strategies may not be directly applicable to our study since we use an intermediate outcome and the ICER was in terms of cost per TB case detected. Another limitation of our study is that the outcome data were collected from secondary sources, and it is impossible to check the accuracy of the test result. Consequently, this can, to some extent, overestimate or underestimate each method's result during diagnosis. However, as our sensitivity analysis shows, the overall ICER's overall effect is likely minimal. Failure of the cost-effectiveness analysis to indicate the affordability of the cost-effective strategy, as we did not conduct budget impact analysis, can also influence the full implementation of results from our study.

\section{Conclusion}

The unit cost of GeneXpert is higher than that of the AFB smear microscopy technique. However, the GeneXpert diagnostic method identified more cases compared to smear microscopy techniques. The use of GeneXpert as a routine test compared to standard care has ICER of $\$ 729.8$ per proportion of cases detected. However, the cost-effectiveness of this technology was influenced by the prevalence of TB. Therefore, the $\mathrm{FMOH}$ and Regional Health Bureau and different stakeholders should consider facilities testing volume, budget, and TB prevalence during the implementation of the diagnostic method as a routine method.

\section{Abbreviations}

AFB

Acid Fast Bacilli; ACER:Average Cost-Effectiveness Ratio; CDR:Case Detection Rate; CEA:CostEffectiveness Analysis; EFY:Ethiopian Birr; EFY:Ethiopian Fiscal Year; EQC:External Quality Control; 
GDP:Gross Domestic Product; FMOH:Federal Ministry of Health; HIV Human Immunodeficiency Virus;

ICER:Incremental Cost-Effectiveness Ratio; LMIC:Low and Medium-Income Countries; MODS:Microscopic Observation Drug Susceptibility; MDR-TB:Multi-Drug Resistant TB; MDR/RIF:Multi-Drug Resistant TB/Rifampicin; MTB:Mycobacterium TB; NTLCP:National TB and Leprosy Control Program; NPV:Negative Predictive Value; ORHB:Oromia Regional Health Bureau; OPHRCBQAL:Oromia Public Health Research Capacity Building \& Quality Assurance Laboratory; PPV:Positive Predictive Value; PSA:Probabilistic Sensitivity Analysis; PTB:Pulmonary TB; POC:Point of Care; QC:Quality Control; SOP:Standard Operating Procedure; TB:TB; TB/HIV:TB/Human immune Virus; TSR:Treatment Success Rate; US\$:United State Dollar; WHO:World Health Organization; YLD:Years Lived with Disability; WTP:Willingness to Pay; ZHD:Zonal Health Department

\section{Declarations}

\section{Ethics approval and consent to participate}

Ethical approval was obtained from the Addis Ababa University School of Public Health Institutional Review Board. The ethical review board provides a waiver for consent to participate since data were collected from patient records.

\section{Consent for publication:}

Not applicable

\section{Availability of data and material:}

The datasets used or analyzed during this study were available from the corresponding author on reasonable request.

\section{Competing interests:}

The authors declare that they have no competing interests

\section{Funding:}

AK was supported by Addis Ababa University, School of Public Health grant. AH was supported by the Bill \& Melinda Gates Foundation grant (OPP1162384). The funders had no role in study design, data collection, and analysis, decision to publish, or preparation of the manuscript.

\section{Authors' contributions}


$\mathrm{AK}$ and $\mathrm{AH}$ designed and worked on the study protocol. AK and $\mathrm{AH}$ prepared a data collection tool and provided training to data collectors. AK and AH conducted a decision tree analysis using TreeAge Pro software. AK and AH analyzed the costs and effectiveness data, interpreted the result, and wrote the draft and final version of the manuscript. Both $\mathrm{AK}$ and $\mathrm{AH}$ read and approved the final manuscript.

\section{References}

1. WHO. Global burden of disease report. In.; 2016.

2. WHO. Global tuberculosis report. In.; 2014.

3. Federal Ministry of Health of Ethiopia. Tuberculosis, Leprosy and TB/HIV Prevention and Control Programme, Fourth edn; 2008.

4. Federal Ministry of Health of Ethiopia. Ethiopian national populationbased Tuberculosis prevalence survey. In. Addis Ababa; 2011: 1-117.

5. Federal Ministry of Health of Ethiopia. Report on national TB/HIV sentinel surveillance. In. Addis Ababa; 2015.

6. Dobler CC. Screening strategies for active tuberculosis: focus on cost-effectiveness. Clinicoecon Outcomes Res. 2016;8:335-47.

7. Brodie D, Schluger NW. The diagnosis of tuberculosis. Clin Chest Med. 2005;26(2):247-71. vi.

8. Churchyard GJ, Stevens WS, Mametja LD, McCarthy KM, Chihota V, Nicol MP, Erasmus LK, Ndjeka NO, Mvusi L, Vassall A, et al: Xpert MTB/RIF versus sputum microscopy as the initial diagnostic test for tuberculosis: a cluster-randomized trial embedded in South African roll-out of Xpert MTB/RIF. Lancet Global Health 2015, 3.

9. Dagnaw AM, BZ T. Laboratory diagnostic systems used in the diagnosis of tuberculosis in Ethiopia: A systematic review. Journal of Medical Laboratory Diagnosis. 2014;5(2):14-21.

10. Piatek AS, Van Cleeff M, Alexander H, Coggin WL, Rehr M, Van Kampen S, Shinnick TM, Mukadi Y. GeneXpert for TB diagnosis: planned and purposeful implementation. Glob Health Sci Pract. 2013;1(1):18-23.

11. Al-Darraji HA, Abd Razak H, Ng KP, Altice FL, Kamarulzaman A. The diagnostic performance of a single GeneXpert MTB/RIF assay in an intensified tuberculosis case finding survey among HIVinfected prisoners in Malaysia. PLoS One. 2013;8(9):e73717.

12. WHO. Global tuberculosis report. In.; 2015.

13. Federal Ministry of Health of Ethiopia. Implementation guideline for GeneXpert MTB/RIF assay in Ethiopia In. Addis Ababa; 2014.

14. Trébucq A, Enarson DA, Chiang CY, Deun AV, Harries AD, Boillot F, Detjen A, Fujiwara PI, Graham SM, Monedero I, et al. Xpert® MTB/RIF for national tuberculosis programmes in low-income countries: when, where and how? International journal of tuberculosis lung disease. 2011;15(12):1567-71.

15. Vassall A, van Kampen S, Sohn H, Michael JS, John KR, den Boon S, Davis JL, Whitelaw A, Nicol MP, Gler MT, et al. Rapid diagnosis of tuberculosis with the Xpert MTB/RIF assay in high burden 
countries: a cost-effectiveness analysis. PLoS Med. 2011;8(11):e1001120.

16. WHO. Automated real-time nucleic acid amplification technology for rapid and simultaneous detection of tuberculosis and rifampicin resistance: Xpert MTB/RIF assay for the diagnosis of pulmonary and extrapulmonary TB in adults and children. 2013.

17. Diagnostics F. Negotiated Product Pricing. In..

18. ST

partnership/GDF. ST: Ensuring an interrupted supply of quality assured, affordable tuberculosis medicines and diagnostic to the world. In. Geneva, Switzerland.; July, 2018.

19. Van Rie A, Page-Shipp L, Hanrahan CF, Schnippel K, Dansey H, Bassett J, Clouse K, Scott L, Stevens W, Sanne I. Point-of-care Xpert(R) MTB/RIF for smear-negative tuberculosis suspects at a primary care clinic in South Africa. Int J Tuberc Lung Dis. 2013;17(3):368-72.

20. Nadia Yakhelef M, Audibert F, Varaine J, Chakaya, Sitienei J: Is introducing rapid culture in the diagnostic algorithm of smear-negative tuberculosis cost-effective? halshs-00866530 2013, 17.

21. National Bank of Ethiopia: Ethiopia: Macroeconomic and Social Indicators. (2018-2019). In.; 2020.

22. Uddin MKM, Chowdhury MR, Ahmed S, Rahman MT, Khatun R, Leth FV. S B: Comparison of direct versus concentrated smear microscopy in the detection of pulmonary tuberculosis. BMC Research Notes 2013, 6(291).

23. Ghiasi M, Pande T, Pai M. Advances in Tuberculosis Diagnostics. Current Tropical Medicine Reports. 2015;2(2):54-61.

24. Pinto M, Entringer AP, Steffen R. A T: Cost analysis of nucleic acid amplification for diagnosing pulmonary tuberculosis, within the context of the Brazilian Unified Health Care System. Journal of Brasil Pneumol. 2015;41(6):536-8.

25. Rupert S, Vassall A, Raizada N, Khaparde SD, Boehme C, Salhotra VS, Sachdeva KS, Nair SA, Hoog $\mathrm{AH}$. Bottom-up or top-down: unit cost estimation of tuberculosis diagnostic tests in India. Int J Tuberc Lung Dis. 2017;21(4):375-80.

26. Maunank Shah V, Chihota G, Coetzee G, Churchyard, Dorman SE. Comparison of laboratory costs of rapid molecular tests and conventional diagnostics for the detection of tuberculosis and drugresistant tuberculosis in South Africa. BMC Infectious Diseases 2013, 13(352).

27. Walusimbi S, Kwesiga B, Rodrigues R, Haile M, de Costa A, Bogg L, Katamba A. Cost-effectiveness analysis of microscopic observation drug susceptibility test versus Xpert MTB/Rif test for diagnosis of pulmonary tuberculosis in HIV patients in Uganda. BMC Health Serv Res. 2016;16(1):563.

28. Pantoja A, Fitzpatrick C, Vassall A, Weyer K, Floyd K. Xpert MTB/RIF for diagnosis of tuberculosis and drug-resistant tuberculosis: a cost and affordability analysis. Eur Respir J. 2013;42(3):708-20.

29. Zwerling AA, Sahu M, Ngwira LG, Khundi M, Harawa T, Corbett EL, Chaisson RE, Dowdy DW. Screening for Tuberculosis Among Adults Newly Diagnosed With HIV in Sub-Saharan Africa: A CostEffectiveness Analysis. J Acquir Immune Defic Syndr. 2015;70(1):83-90. 
30. Hsiang E, Little KM, Haguma P, Hanrahan CF, Katamba A, Cattamanchi A, Davis JL, Vassall A, Dowdy D. Higher cost of implementing Xpert((R)) MTB/RIF in Ugandan peripheral settings: implications for cost-effectiveness. Int J Tuberc Lung Dis. 2016;20(9):1212-8.

31. Teran R, JH W. Recent advances in the laboratory diagnosis of tuberculosis. International federation of clinical chemistry laboratory medicine. 2015;26(4):295-309.

32. Wang G, Wang S, Jiang G, Fu Y, Shang Y, Huang H. Incremental cost-effectiveness of the second Xpert MTB/RIF assay to detect Mycobacterium tuberculosis. J Thorac Dis. 2018;10(3):1689-95.

33. Cowan JF, Chandler AS, Kracen E, Park DR, Wallis CK, Liu E, Song C, Persing DH, Fang FC. Clinical Impact and Cost-effectiveness of Xpert MTB/RIF Testing in Hospitalized Patients With Presumptive Pulmonary Tuberculosis in the United States. Clin Infect Dis. 2017;64(4):482-9.

34. Jha S, Ismail N, Clark D, Lewis JJ, Omar S, Dreyer A, Chihota V, Churchyard G, Dowdy DW. CostEffectiveness of Automated Digital Microscopy for Diagnosis of Active Tuberculosis. PLoS One. 2016;11(6):e0157554.

35. Adelman MW, McFarland DA, Tsegaye M, Aseffa A, Kempker RR. HM B: Cost-effectiveness of WHORecommended Algorithms for TB Case Finding at Ethiopian HIV Clinics. Infectious disease society of America 2017.

36. Andrews JR, Lawn SD, Rusu C, Wood R, Noubary F, Bender MA, Horsburgh CR, Losina E, Freedberg KA, Walensky RP. The cost-effectiveness of routine tuberculosis screening with Xpert MTB/RIF prior to initiation of antiretroviral therapy: a model-based analysis. AIDS. 2012;26(8):987-95.

37. Choi HW, Miele K, Dowdy D. M S: Cost-effectiveness of Xpert ${ }^{\circledR}$ MTB/RIF for diagnosing pulmonary tuberculosis in the United States. International Journal of Tuberculosis Lung Disease. 2013;17(10):1328-35.

38. Dowdy DW, Steingart KR, Pai M. Serological testing versus other strategies for diagnosis of active tuberculosis in India: a cost-effectiveness analysis. PLoS Med. 2011;8(8):e1001074.

39. James A, Ochei K, Emenyonu N. L L: Effectiveness of Tuberculosis Smear Microscopy in Laboratory Diagnosis of Mycobacterium tuberculosis in Resource-Constrained Settings. British Journal of Medicine Medical Research. 2015;9(11):1-8.

40. Opota O, Senn L, Prod'hom G, Mazza-Stalder J, Tissot F, Greub G, Jaton K. Added value of molecular assay Xpert MTB/RIF compared to sputum smear microscopy to assess the risk of tuberculosis transmission in a low-prevalence country. Clin Microbiol Infect. 2016;22(7):613-9.

41. WHO. Global tuberculosis report. In.; 2018.

\section{Figures}




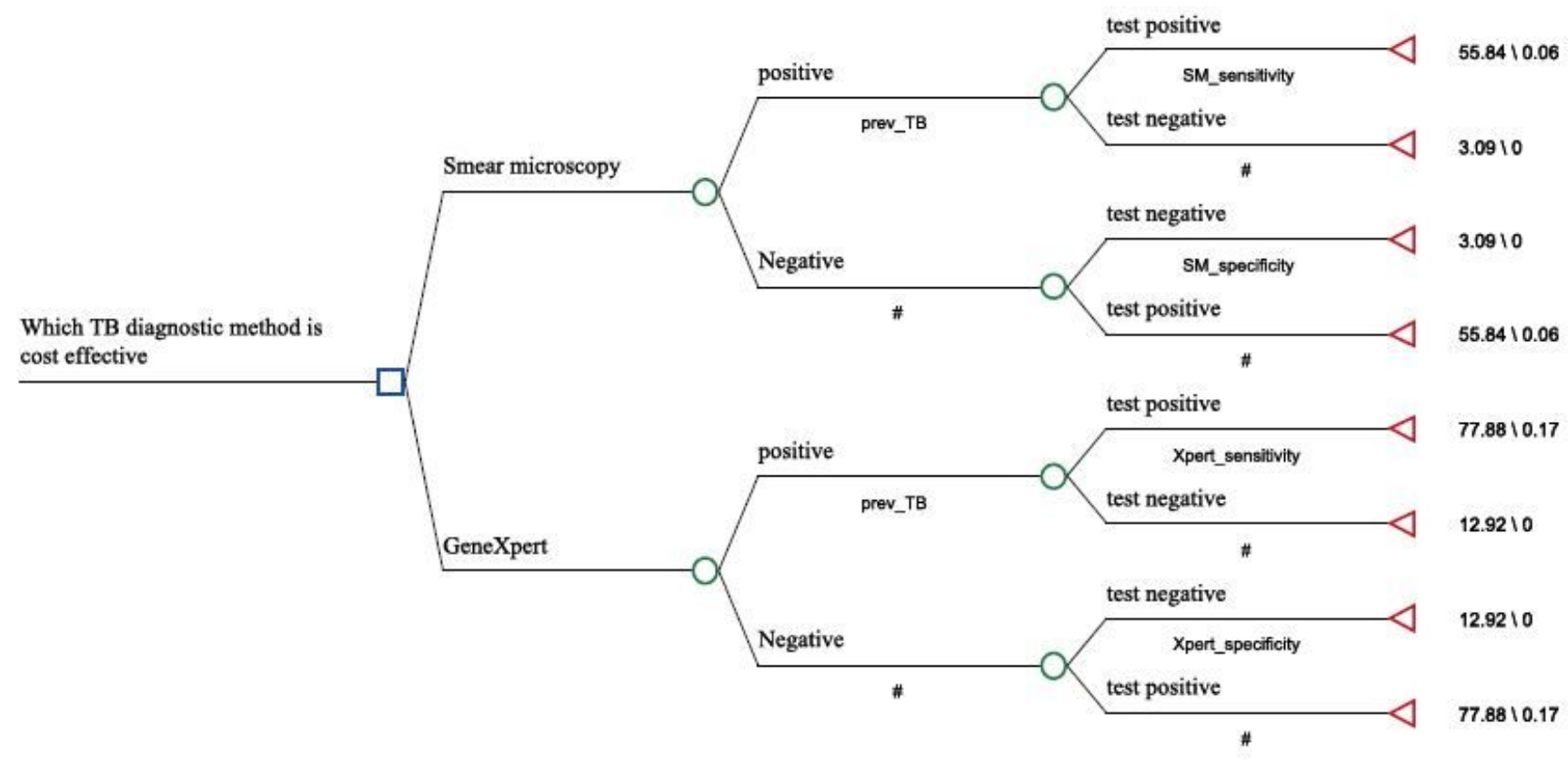

\section{Figure 1}

Decision tree analysis model for TB diagnostic methods

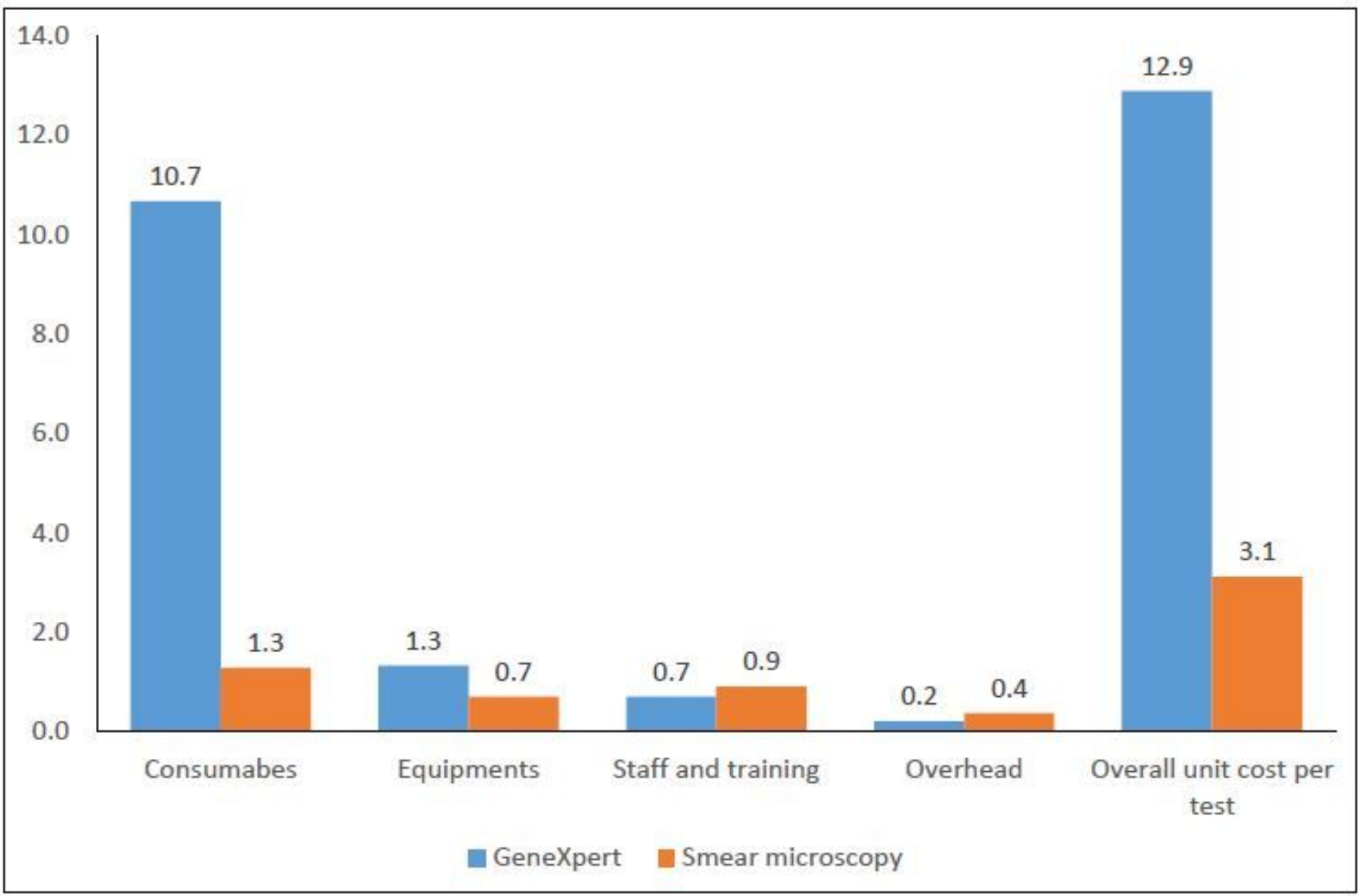


Figure 2

Unit cost per test for smear microscopy versus GeneXpert diagnostic methods

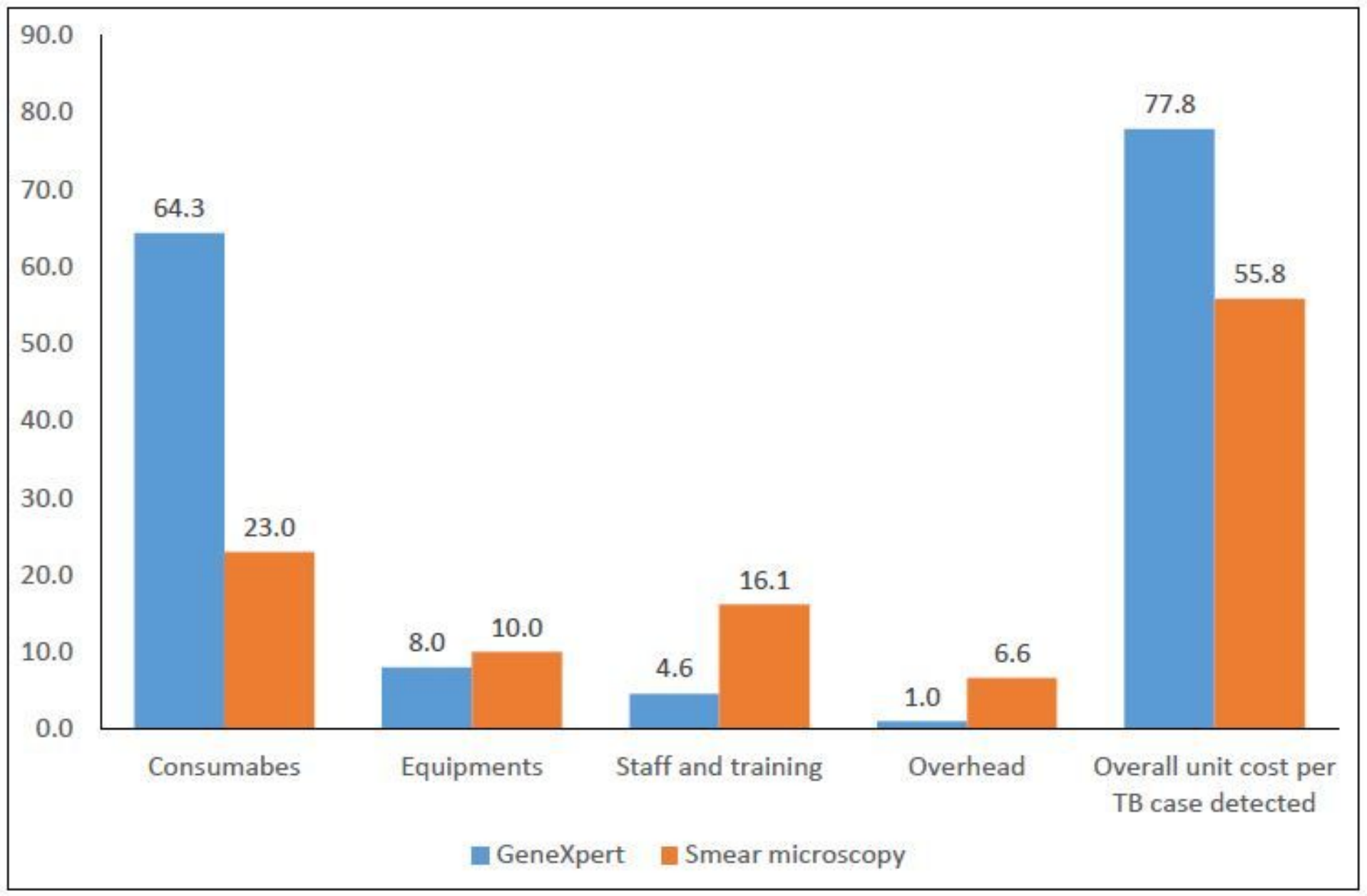

\section{Figure 3}

Unit cost per TB case detected for smear microscopy versus GeneXpert diagnostic methods Scenario analysis of the costs 


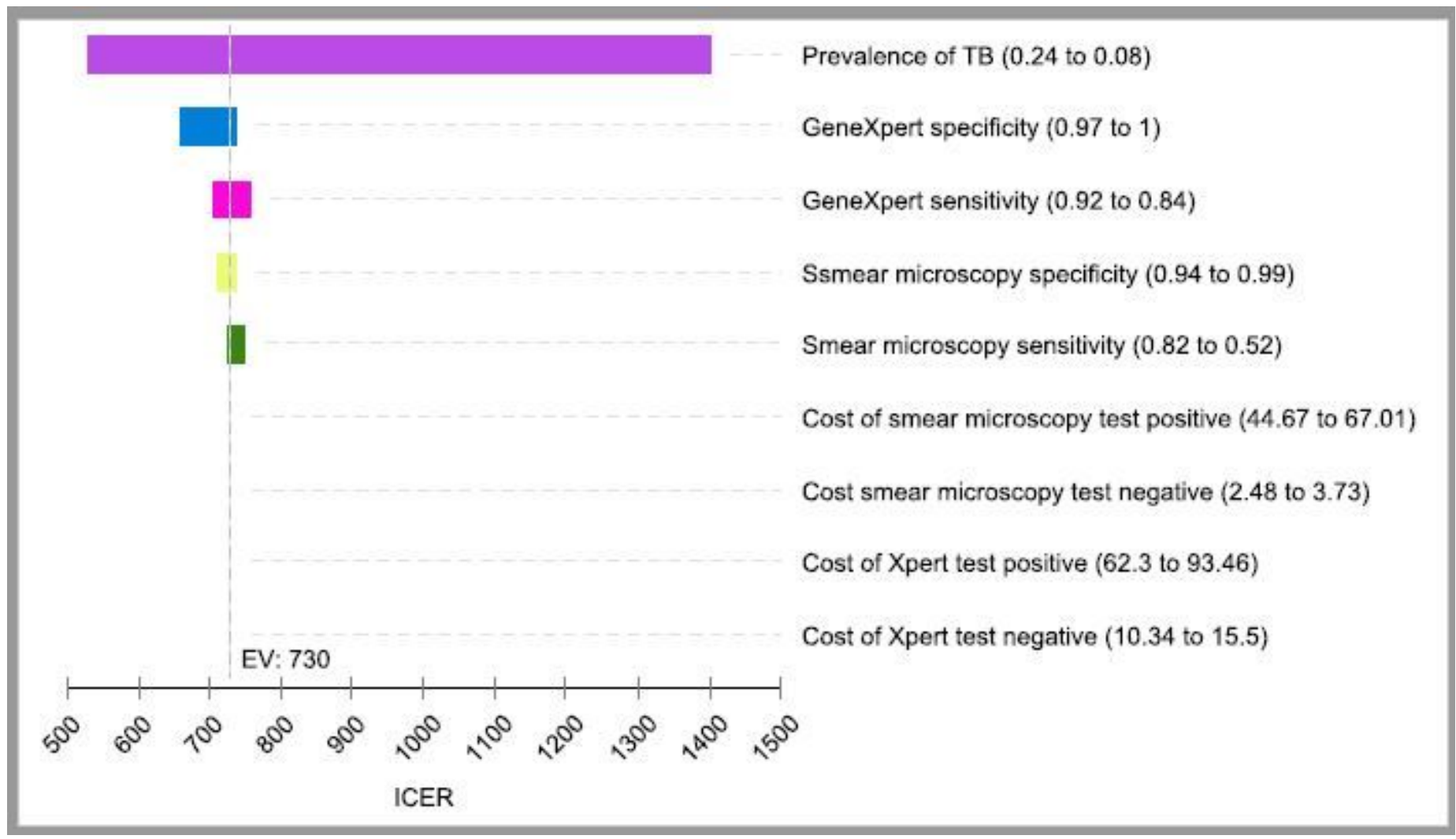

\section{Figure 4}

One-way sensitivity analysis of ICER comparing GeneXpert to smear microscopy method using tornado diagram.

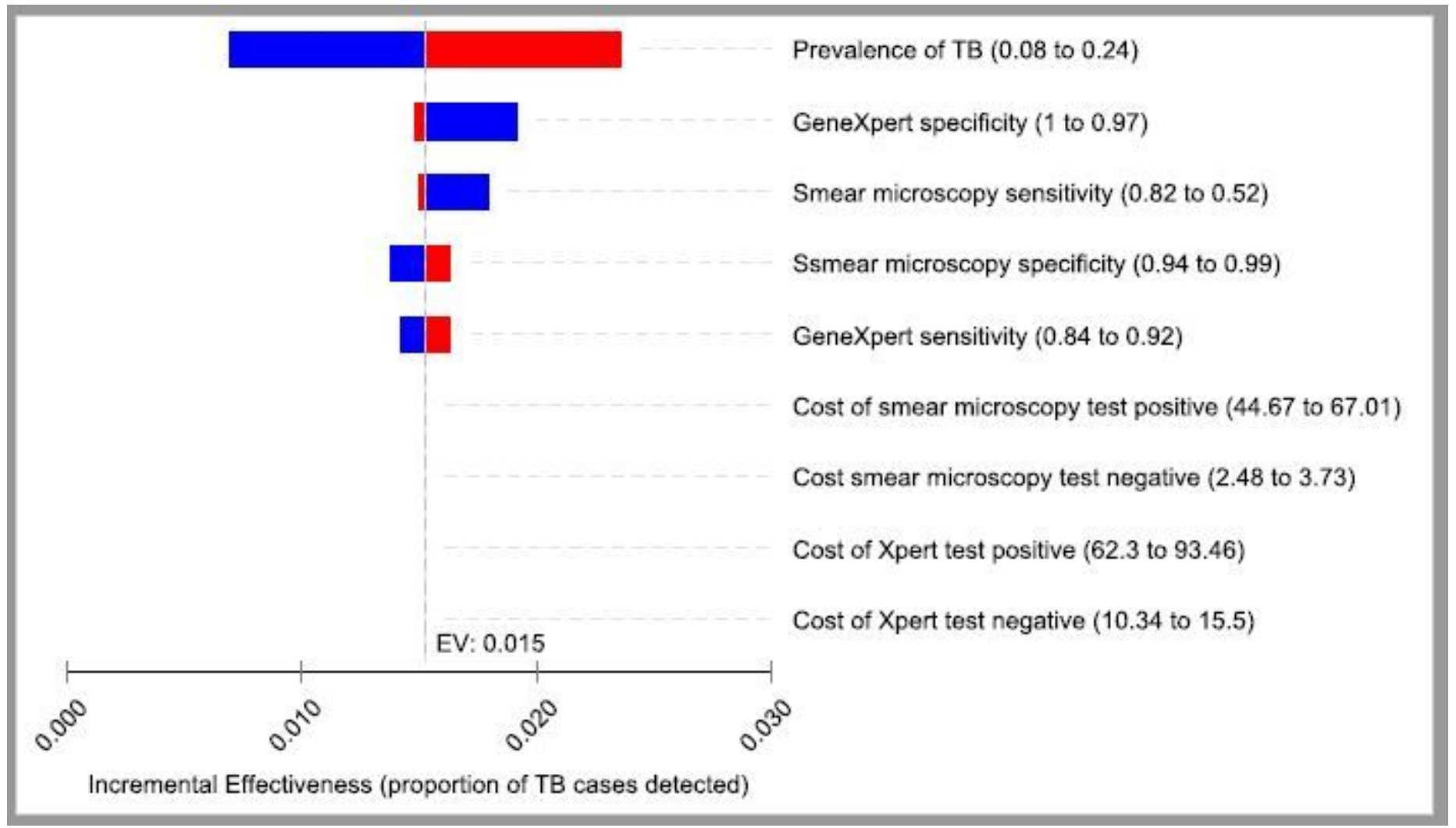


One-way sensitivity analysis of incremental proportion case detected comparing the GeneXpert Algorithm to smear microscopy

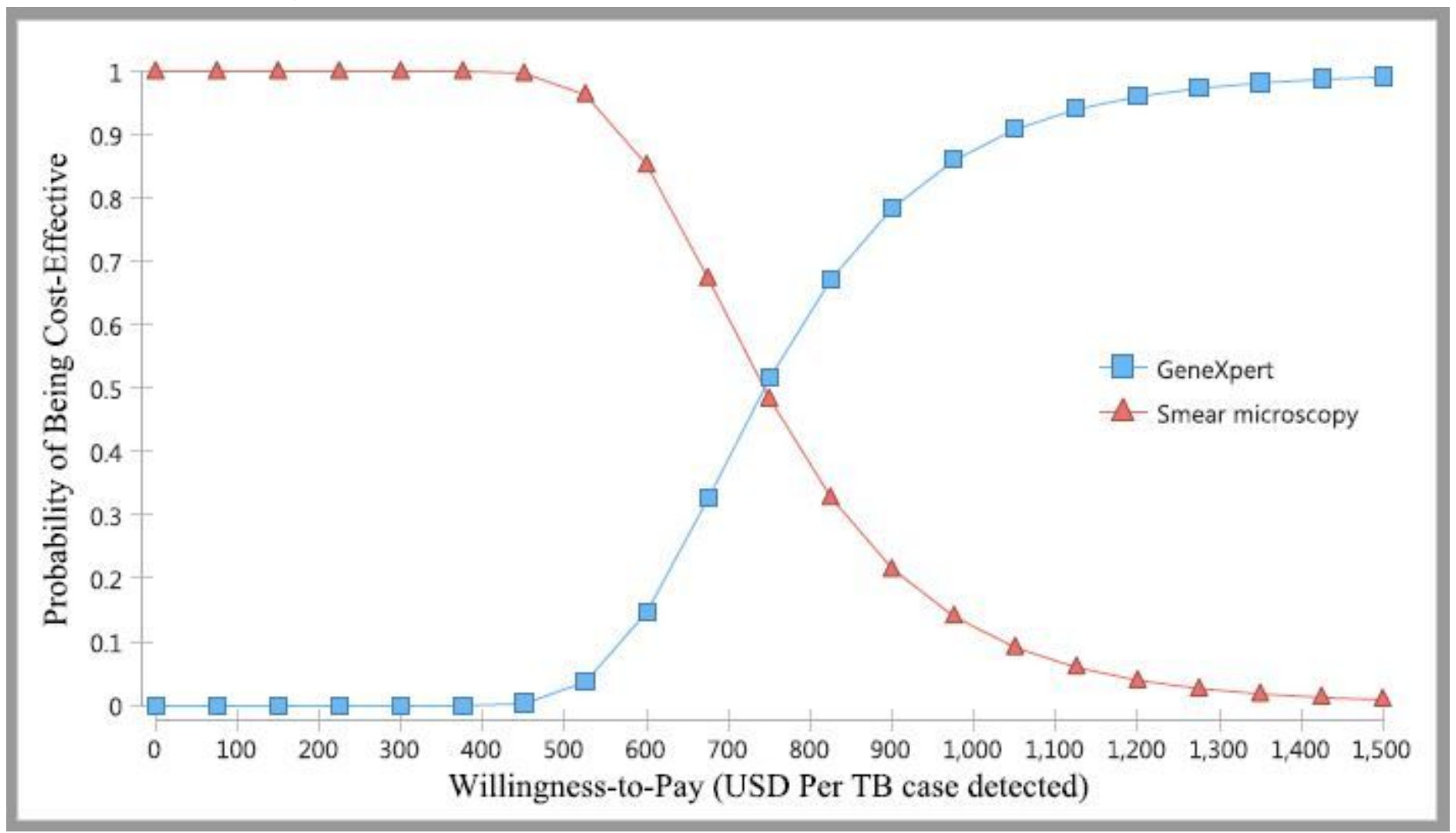

\section{Figure 6}

Cost-effectiveness acceptability curve comparing GeneXert and smear microscopy technique 